\title{
TEMPORARY SET, AS ASSOCIATED WITH MAGNETOSTRICTION.
}

\author{
By CARl Barus.
}

\section{INTRODUCTION.}

$\mathrm{I}$ my earlier papers ${ }^{1}$ on the subject, I inferred that the brilliant theories of magnetostriction due to Kirchoff, Korteweg, Lorberg, Helmholtz, Maxwell and others, can have but a limited application, inasmuch as the phenomena are inherently associated with what I venture to interpret as viscosity. In other words, if deformations evoked by physical changes of molecular configuration within the metal be termed viscous, whether gradual or sudden, then viscosity enters essentially into magnetostriction. With each twist, however small, and within the elastic limits the wire receives a certain amount of set, instantaneously; the whole of this set may be shaken out by the first magnetization and it is thus appropriately called temporary, to distinguish it from permanent set which comes much later, as a second phase lying beyond the limits of elasticity. On removing the magnetic field, therefore, the unmagnetic wire is molecularly different from the same unmagnetic wire before magnetization; and the new molecular arrangement is permanent so long as the twist is unchanged. Any change of twist, whether increment or decrement evokes new temporary set, or a new molecular grouping, which may be converted into the permanent grouping for the given twist, by a reapplication of the magnetic shake up in question. Hence throughout the whole period within which varying torsional stresses are applied, the metal is undergoing continual change of molecular constitution, which in relation to magnetostriction are as radical as though a new metal ${ }^{2}$ were being continu-

1 American Journal of Science, XXXIV., p. 175, 1887; X., p. 407, 1900; XI., p. 97, I90I.

${ }^{2}$ One may argue that the metal is not again normal until after the first magnetization. This is what I have done, virtually, and it works well : but one must acknowledge the ground debatable. At all events no fundamentally correct or physically acceptable theory will overlook the temporary set, which is just as much a part of magnetostriction as the elastic phenomena which follow. 
ally supplied for investigation. These are the occurrences which a purely elastic theory does not adequately construe.

The gradual viscous deformations due to the heat motion within the wire are without bearing on the present experiments. They are of a different nature and easily eliminated.

The phenomena may then be analyzed as follows : Let a soft iron wire be subject to a fixed amount of torque, corresponding to a fixed amount of twist registered as a definite deflection (fiducial zero) on some appropriate scale. The first magnetization will produce (if twist is large enough) an increment of rigidity, registered as an increased deflection (say) on the scale. The withdrawal of the field does not reproduce the original deflection; the new deflection is in all cases smaller than the original deflection or below the fiducial zero; i. e., the unmagnetic wire was virtually more rigid before than after the magnetization. If torque remains constant, the new conditions are persistent; so that on subsequent magnetizations the second deflection, on subsequent demagnetizations the third deflection, is regained almost perfectly. The inelastic feature has now been eliminated for the particular torque impressed. As the second deflection is eventually an increment, the third a decrement on the first, the elastic phenomenon is the numerical sum of both and is thus greater than either. There has been a slip of the fiducial zero favorable to greater deflection.

2. Plan and Purpose.-In the papers mentioned, more particularly in the last, I paid considerable attention to temporary set or slip as I then called it. It was found to be not very different (relatively) in the smallest $\left(45^{\circ}\right)$ and in the largest $\left(360^{\circ}\right)$ twists applied. Indeed between these limits of impressed stress, it seemed to pass through a maximum. Obviously, however, the slip must initially increase from zero with the twist. It is thus the chief purpose of the present paper to investigate the character and occurrence of temporary set for twists smaller than $45^{\circ}$, indefinitely. I shall moreover use a slightly different method of discussion, separating the increment of rigidity due to the first magnetization from the decrement of rigidity (slip) due to the first demagnetization for the purpose of discerning the nature of each.

In the interest of a definite scheme of investigation, the twists im- 
parted should in some way regularly increase toward, or decrease from the highest values admissible. This is best accomplished by twisting the wire back and forth by amounts increasing in fixed steps and lying symmetrically on both sides of zero. In such a case the antecedent twist, i. e., the total strain applied in any one direction since the last magnetization, will be the numerical sum of the positive twist and the negative twist from which the positive twist has been imparted; and vice versa. In other words, temporary set will here depend on an angle different from the impressed or actuating twist, while the increment of rigidity depends on the latter.

Again, if the wire is twisted in regularly increasing steps on one side of zero only, it should be brought back to zero for investigation between each twist. In this case the antecedent twist which determines the temporary set is the twist last impressed, while the actuating twist is zero.

What I make clear in this paper, is the dependence of temporary set or slip on the antecedent strain as above defined. Its direction is immaterial so far as the numerical amount of slip is concerned. The conditions under which slip is picked up by the magnetically increased rigidity in the presence of an impressed strain will also appear.

Throughout this paper in referring to magnetically increased rigidity I have in mind the simple torsion model described in an earlier paper. ${ }^{1}$ When the wire is twisted the increment of tension along the lines of longitudinal stress due to magnetization, acts with a horizontal component in a direction equivalent to an increase of rigidity.

3. Apparatus.-This was the same differential apparatus heretofore described, consisting of identical upper and lower, collinear, vertical soft iron wires, joined in the middle by a light brass mirror support, and fastened at the ends to torsion heads. The upper wire was surrounded by a helix with internal water circulation. The two wires are countertwisted by the amounts $\theta$ each as given in the tables. The deflections occurring at the mirror when one wire is acted on magnetically, are observed on a centimeter scale 6 me-

1 American Journ., X., p. 40I, r9oo. 
ters distant. Thus $2 \mathrm{I} \mathrm{cm}$. of deflection correspond to one degree. The wires were each usually $37 \mathrm{~cm}$. long and $.044 \mathrm{~cm}$. in diameter. The magnetic field of the long helix may be rated at about 100 c.g.s units per ampere.

The system of two wires was kept under a definite pull of about 300 grams, an insignificant amount. If the upper wire is twisted clockwise to an eye regarding it from above, an increased deflection denotes virtually increased rigidity of the upper (magnetized) wire.

This differential method was devised as has been stated elsewhere, to obtain direct relations between the deflection increments and the corresponding changes of rigidity. For if $\theta$ be the twist imparted to each wire, $n$ their common original rigidity, if $d \theta$ and $d n$ be the changes due to magnetostriction, then $n(\theta+d \theta)=(n+d n)(\theta-d \theta)$, seeing that the twist of the upper wire decreases by the same amount as the twist of the lower wire increases, while torque, length and section remain unchanged. Hence

$$
d n / n=2 d \theta / \theta=\mu,
$$

which I have called the coefficient of magnetically increased rigidity $^{1}$. Its value is a definite minimum when the (elastic) obliquity of the external fiber is a maximum.

Results for Successively Increasing Alternating Twists.

4. Preliminary.-These results are given in the following tables, in which the first column shows the twist applied to each wire. The second to fifth columns show the reading at the scale in centimeters, originally (off (I)), and when the current is successively made and broken as the subscripts indicate. The sixth column gives the value of the antecedent twist, being the numerical sum of the existing and the preceding (opposite) twist. Great care must be taken not to exceed the strain limits stated, as otherwise the values of the antecedent twists stated would be quite erroneous. Accidents are noted. The 7 th and 8 th columns show the deflections from the original zero-off ( I) - due to the first magnetization (on), and the first removal of the field. When these deflections have the same sign as the twist the rigidity of the magnetized wire

${ }^{1}$ American Journ., XXXIV., I887, p. I8r et seq. 
TABLE I.

Twists allernating symmetrically. Magnetic field, 380 c.g.s. units. Soft iron; diameter $.044 \mathrm{~cm}$., length $37 \mathrm{~cm}$., scale distance $600 \mathrm{~cm}$., deflection $21 \mathrm{~cm}$., equivalent to $1^{\circ}$.

\begin{tabular}{|c|c|c|c|c|c|c|c|c|}
\hline \multirow{3}{*}{$\begin{array}{l}\text { Twist } \\
\text { of each } \\
\text { Wire. }\end{array}$} & \multicolumn{4}{|c|}{ Scale Reading when Field is } & \multirow{3}{*}{$\begin{array}{l}\text { Ante- } \\
\text { cedent } \\
\text { Strain. }\end{array}$} & \multicolumn{3}{|c|}{ Deflections ${ }^{1}$ Referred to } \\
\hline & \multirow{2}{*}{ Off $(x)$} & \multirow{2}{*}{ On $(x)$} & \multirow{2}{*}{ Off $(2)$} & \multirow{2}{*}{ On $(2)$} & & \multicolumn{2}{|c|}{ Original Zero. } & \multirow{2}{*}{$\frac{\text { New Zero. }}{\text { Total. }}$} \\
\hline & & & & & & On. & Off. & \\
\hline$\circ$ & $\mathrm{cm}$. & $\mathrm{cm}$ & $\mathrm{cm}$ & $\mathrm{cm}$. & $\circ$ & $\mathrm{cm}$ & $\mathrm{cm}$ & $\mathrm{cm}$. \\
\hline-5 & 0 & +1.0 & +1.5 & +1.0 & 5 & -1 & $\overline{-1.5}$ & .5 \\
\hline+5 & 0 & -.5 & -1.0 & -1.5 & 10 & -.5 & -1 & .5 \\
\hline-10 & 0 & -.5 & +1.0 & 0.0 & 15 & .5 & -1 & 1.5 \\
\hline+10 & 0 & -.5 & -1.0 & -.5 & 20 & -.5 & 1 & .5 \\
\hline-20 & 0 & .0 & +1.8 & .0 & 30 & 0 & -1.8 & 1.8 \\
\hline+20 & 0 & -.6 & -2.2 & -.6 & 40 & -.6 & -2.2 & 1.6 \\
\hline-30 & 0 & -.4 & +2.6 & -.4 & 50 & .4 & -2.6 & 3.0 \\
\hline+30 & 0 & .0 & -2.4 & .2 & 60 & 0 & -2.4 & 2.4 \\
\hline-40 & 0 & -.9 & +3.0 & -.8 & 70 & .9 & -3.0 & 3.9 \\
\hline+40 & 0 & +.6 & -2.8 & +.6 & 80 & .6 & -2.8 & 3.4 \\
\hline-50 & 0 & -1.6 & +2.6 & -1.6 & 90 & 1.6 & -2.6 & 4.2 \\
\hline+50 & 0 & +1.4 & +3.1 & +1.4 & 100 & 1.4 & -3.1 & 4.5 \\
\hline 0 & - & - & $\ldots$ & - & - & $\longrightarrow$ & - & - \\
\hline-50 & 0 & -2.6 & 2.2 & 2.4 & 50 & 2.6 & -2.2 & 4.8 \\
\hline+50 & 0 & 1.6 & -2.6 & 1.6 & 100 & 1.6 & -2.6 & 4.2 \\
\hline-60 & 0 & -3.0 & 2.3 & -2.8 & 110 & 3.0 & -2.3 & 5.3 \\
\hline+60 & 0 & 2.2 & -2.9 & 2.2 & 120 & 2.2 & -2.9 & 5.1 \\
\hline-50 & 0 & -1.4 & 2.9 & -1.4 & 110 & 1.4 & -2.9 & 4.3 \\
\hline+50 & 0 & 2.0 & -2.6 & 2.0 & 100 & 2.0 & -2.6 & 4.6 \\
\hline-40 & 0 & -.4 & 3.2 & -.4 & 90 & .4 & -3.2 & 3.6 \\
\hline+40 & 0 & .8 & -2.8 & .8 & 80 & .8 & -2.8 & 3.6 \\
\hline-30 & 0 & .4 & 3.1 & .4 & 70 & -.4 & -3.1 & 2.7 \\
\hline+30 & 0 & .0 & -2.6 & .1 & 60 & .0 & -2.6 & 2.6 \\
\hline-20 & 0 & 1.0 & 2.7 & 1.0 & 50 & -.1 & -2.7 & 1.7 \\
\hline+20 & 0 & .0 & -2.2 & -.2 & 40 & .0 & -2.2 & 2.2 \\
\hline-10 & 0 & 1.0 & 2.0 & 1.0 & 30 & -1.0 & -2.0 & 1.0 \\
\hline+10 & 0 & -.4 & -1.2 & -.3 & 20 & -.4 & -1.2 & .8 \\
\hline-5 & 0 & .7 & 1.0 & .8 & 15 & -.7 & -1.0 & .3 \\
\hline+5 & 0 & -.1 & -.6 & -.1 & 10 & -.1 & -.6 & .5 \\
\hline 0 & 0 & .5 & .5 & .5 & 5 & -.5 & -.5 & .0 \\
\hline
\end{tabular}

is increased; for the case of opposite signs rigidity is decreased. The last column (numerical sum of the preceding two), shows the persistent or elastic increment of rigidity in the wire molecularly modified for the particular twist in question, as explained in paragraph I.

${ }^{1}$ Taking the strains all positive, the deflections are changed in sign accordingly. 
5. Results.-Omitting introductory experiments, results of a definite character are given in table I, though the first few deflections taken but to centimeters, are too crude.

In the accompanying chart, Fig. I, the deflections of the table are mapped out as ordinates relative to the given antecedent strains as abscissas, except in curve 3, where the impressed strains are the abscissas. The impressed strains are marked in degrees of arc on the peaks of the curves.

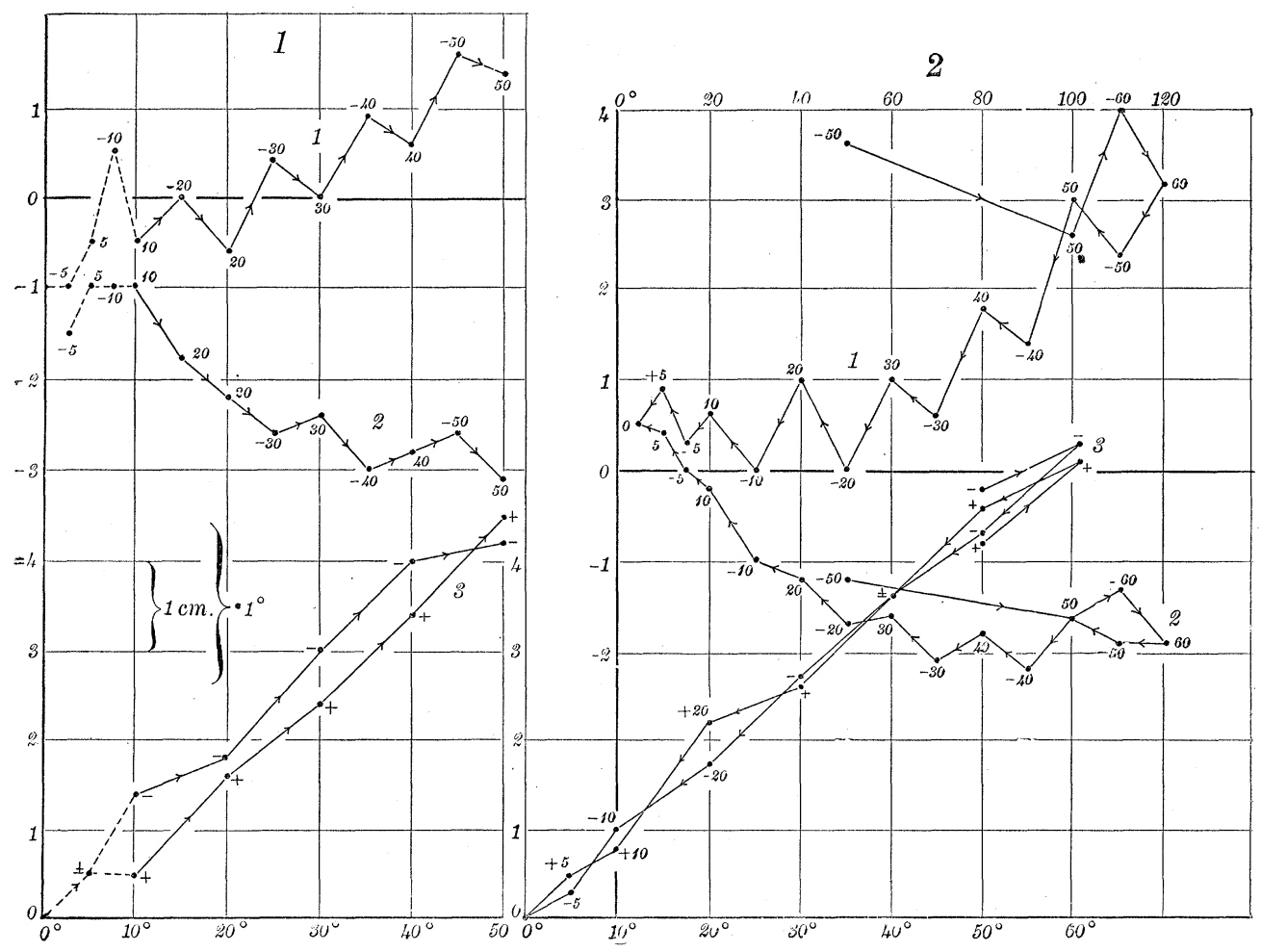

Fig. 1.

Fig. 2.

Curve I denotes the increase of rigidity due to the first magnetization relatively to the zero of the original unmagnetic wire. It shows the effect of temporary set or slip superposed on the effect of magnetically increased rigidity in the presence of the magnetic field and of the impressed strain stated. This curve is distinctly 
alternating in character, like the twists. The rigidity is always greater for the negative twists with which the experiments begin, than for the positive twists of the same character which are subsequent.

As a whole, the mean increase of rigidity beginning with negative values is accelerated as twist increases. There is indication of a minimum.

Curve 2 shows the displacement (slip) of the fiducial zero of the unmagnetic metal before and after the first magnetization. It indicates the effect of slip in the presence of the impressed strain stated and of the magnetization, being the temporary set under these circumstances. Curve 2 corresponds definitely to a decrement of rigidity, which increases numerically with the twist but soon reaches a maximum at about $\pm 30^{\circ}$ or $\pm 40^{\circ}$ of impressed strain. The curve is much smoother than the preceding though it eventually runs into marked alternations. The initial slope is about

$$
d \theta / \theta=.0026 .
$$

Curve 2 in connection with curve I shows that whereas the effect of slip is in excess for small strains, it is soon overtaken by the magnetically increased rigidity. The alternations of both curves correspond in the main, but are opposed in phases.

Curve 3 , the algebraic difference of the two curves, shows the persistent magnetic increase of rigidity after the first magnetization. It represents the amount of magnetically increased rigidity in the absence of slip or temporary set for the impressed strain stated. The mean increment of rigidity is initially $d n / n=2 d \theta / \theta=.008$ for the positive twists and .0096 for the negative twists.

The second part of Table II. and the accompany chart (Fig. 2) contain corresponding results for a twist decreasing from $120^{\circ}$ in successive steps of $10^{\circ}$. The curves are numbered as in the preceding chart.

Curve I has similar alternating characteristics to the preceding case; but the results are clearer throughout and they substantiate the occurrence of a minimum in the mean values for impressed twists of about $\pm 10^{\circ}$ or $\pm 20^{\circ}$. Beyond this, however, the curve presents a new result; the rigidities of the positive twists 
are now successively in excess over the negative. The change of sign occurs at $+60^{\circ}$ at the end of the curve.

TABLE II.

Twists alternating symmetrically. Magnetic field, 720 c.g.s. Soft iron, diameter .044, length $37 \mathrm{~cm}$. Scale distance $600 \mathrm{~cm}$. Deflection, $21^{\circ} \mathrm{cm}$. equivalent to 1.

\begin{tabular}{|c|c|c|c|c|c|c|c|c|}
\hline \multirow{3}{*}{$\begin{array}{l}\text { Twist } \\
\text { of Each } \\
\text { Wire. }\end{array}$} & \multicolumn{4}{|c|}{ Scale Reading for Field. } & \multirow{3}{*}{$\begin{array}{c}\text { Ante- } \\
\text { cedent } \\
\text { Strain. }\end{array}$} & \multicolumn{3}{|c|}{ Deflections ${ }^{1}$ Referred to } \\
\hline & \multirow{2}{*}{ Off $(x)$} & \multirow{2}{*}{ On $(x)$} & \multirow{2}{*}{ Off $(2)$} & \multirow{2}{*}{ On $(2)$} & & \multicolumn{2}{|c|}{ Original Zero. } & \multirow{2}{*}{$\frac{\text { New Zero }}{\text { Total. }}$} \\
\hline & & & & & & On & Off. & \\
\hline$\circ$ & $\mathrm{cm}$. & $\mathrm{cm}$ & $\mathrm{cm}$. & $\mathrm{cm}$ & $\circ$ & $\mathrm{cm}$ & $\mathrm{cm}$ & $\mathrm{cm}$. \\
\hline 0 & & - & & $\longrightarrow$ & 0 & - & - & - \\
\hline-5 & 0 & -.2 & .2 & -.4 & 5 & .2 & -.2 & .4 \\
\hline+5 & 0 & 0 & .8 & .0 & 10 & 0 & -.8 & .8 \\
\hline-10 & 0 & -.2 & 1.0 & -.2 & 15 & .2 & -1.0 & 1.2 \\
\hline+10 & 0 & -.4 & -1.6 & -.4 & 20 & -.4 & -1.6 & 1.2 \\
\hline-20 & 0 & -.2 & 1.9 & 0 & 30 & .2 & -1.9 & 2.1 \\
\hline+20 & 0 & -.2 & -2.8 & -.2 & 40 & -.2 & -2.8 & 2.6 \\
\hline-30 & 0 & -.8 & 2.4 & -.8 & 50 & .8 & -2.4 & 3.2 \\
\hline+30 & 0 & -.2 & -3.2 & .2 & 60 & .2 & -3.2 & 3.4 \\
\hline-40 & 0 & -1.2 & 3.0 & -1.1 & 70 & 1.2 & -3.0 & 4.2 \\
\hline+40 & 0 & 1.0 & -3.1 & 1.0 & 80 & 1.0 & -3.1 & 4.1 \\
\hline-50 & 0 & -2.8 & 2.2 & 2.8 & 90 & 2.8 & -2.2 & 5.0 \\
\hline+50 & 0 & -2.0 & -3.0 & 2.0 & 100 & 2.0 & -3.0 & 5.0 \\
\hline-40 & 0 & -1.4 & 2.6 & -1.3 & 90 & 1.4 & -2.6 & 4.0 \\
\hline+40 & 0 & 1.0 & -2.9 & 1.0 & 80 & 1.0 & -2.9 & 3.9 \\
\hline-30 & 0. & .2 & 3.3 & .2 & 70 & -.2 & -3.3 & 3.1 \\
\hline+30 & 0 & .1 & -3.1 & .1 & 60 & .1 & -3.1 & 3.2 \\
\hline-20 & 0 & .8 & 2.9 & 1.0 & 50 & -.8 & -2.9 & 2.1 \\
\hline+20 & 0 & -.4 & -3.0 & .4 & 40 & -.4 & -3.0 & 2.6 \\
\hline-10 & 0 & 1.0 & 2.2 & 1.0 & 30 & -1.0 & -2.2 & 1.2 \\
\hline+10 & 0 & -.2 & -1.6 & -.2 & 20 & -.2 & -1.6 & 1.4 \\
\hline-5 & 0 & 1.0 & 1.2 & 1.0 & 15 & -1.0 & -1.2 & .2 \\
\hline+5 & 0 & .6 & -.4 & .6 & 10 & .6 & -.4 & 1.0 \\
\hline 0 & 0 & $.5^{2}$ & .2 & .5 & 5 & .5 & .2 & .7 \\
\hline
\end{tabular}

Curve 2 showing the temporary set under the given conditions is again a much smoother curve initially, indicating a decrement of rigidity with a limit reached at about $\pm 40^{\circ}$. Relatively to curve $\mathrm{I}$, slip is initially in excess, magnetically incremented rigidity even-

${ }^{1}$ Strains taken positive and signs of deflections changed accordingly.

${ }^{2}$ Zero at $-7^{\circ}$, due to permanent set. 
tually so, as before. The alternations of curves I and 2 correspond, but are now in the same phase. The initial slope is

$$
d \theta / \theta=.003 .
$$

Curve 3 finally oscillates in irregular fashion about a straight line such that $d n / n=2 d \theta / \theta=.0088$, remembering that the abscissas here are actuating twists and not antecedent strains.

6. Repetition of a Complete Cycle.-The following cycles alternate in steps of $10^{\circ}$ as far as $\pm 50^{\circ}$, but a stronger field of about 700 c.g.s. units is brought to bear. As a result the helix became warm, and gave rise to a permanent set of about $7^{\circ}$, the zero being found at $-7^{\circ}$ on unlocking the instrument, after completing the retrograde march.

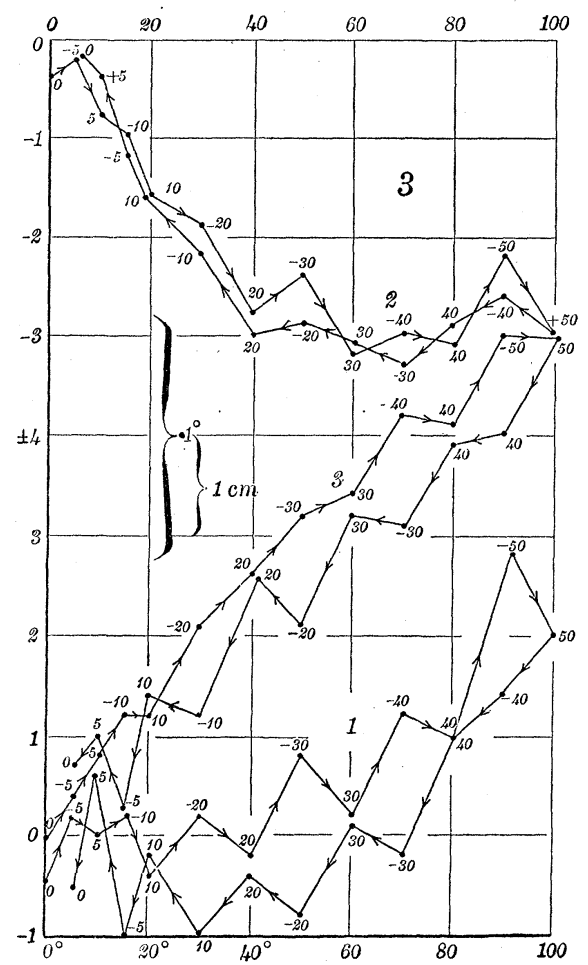

Fig. 3.

The results are given in Table II., following and constructed in in the chart Fig. 3, in the usual way. Curve I shows the effect of 
the first magnetization referred to the original molecular status of the non-magnetic wire. Arrows indicate the progress of the experiment, which begins and ends at zero. The features of the preceding observations definitely reappear. The curve alternates with the twist; its mean value increases with the twist at an accelerated rate, passing from negative values, through a minimum at about $10^{\circ}$; in the outgoing march, the rigidity for the negative twists is greater than the rigidity of the corresponding positive twists; the reverse holds for the return curve. The curve of temporary set, 2 , is again decremental in its two branches, and the march is definitely toward a limiting value at about $\pm 30^{\circ}$ or $\pm 40^{\circ}$. It is smoother at first but oscillates markedly in the region of higher twists. The slope $d \theta / \theta=.0033$.

Finally both branches of curve 3 , which shows the magnetically increased rigidity relatively to the new molecular status of the unmagnetic wire, are here given in terms of the antecedent strains as abscissas, merely for the sake of a clearer location of the observations. Relatively to the impressed twists (not shown) both branches may be described as oscillating irregularly about a straight line such that the corresponding elastic increment of rigidity is

$$
d n / n=2 d \theta / \theta=.010 .
$$

The effect of doubling the magnetic field is thus not marked, for the slopes above (.0096 and .0088) are not much smaller, while the field was about half as large. Saturation may be assumed for both cases.

7. Remarks in Explanation.-The inferences of my earlier papers (1. c.) will be seen to be corroborated throughout and need not therefore be repeated. What is new, viz., the growth of different phases of the phenomena within $\pm 50^{\circ}$, has also been fully presented. With regard to slip, which reaches a limit at say $\pm 40^{\circ}$ in the presence of impressed strain, it appears that after passing this value temporary set is more and more fully removed by the direct effect of magnetization, finally to fall off indefinitely.

The character of the curves I, or the deflection produced on first magnetizing the non-magnetic metal in its original molecular status for the given twist, remains for explanation. The features of this 
curve are its alternations, which are larger for a negative twist than for the succeeding positive twist of the same value in the outgoing series, with the reversed result in the return series; or vice versa. The peaks of the curves are of different sign in the advance and the retrogression, respectively. This observation is of considerable importance in its bearing on the present phenomena as will be seen in the sequel.

To account for the result it is at the outset sufficient to recognize (as already intimated) that the amount of temporary set or slip depends on the antecedent strain, whereas the increment of rigidity, caet. par., depends on the impressed strain or stress actually in action. Hence if a wire is twisted alternately in steps of $\pm \theta$ to a limit, and in like manner untwisted, the following series will determine the slip and the advance (incremented rigidity) due to the first magnetization of the original metal.
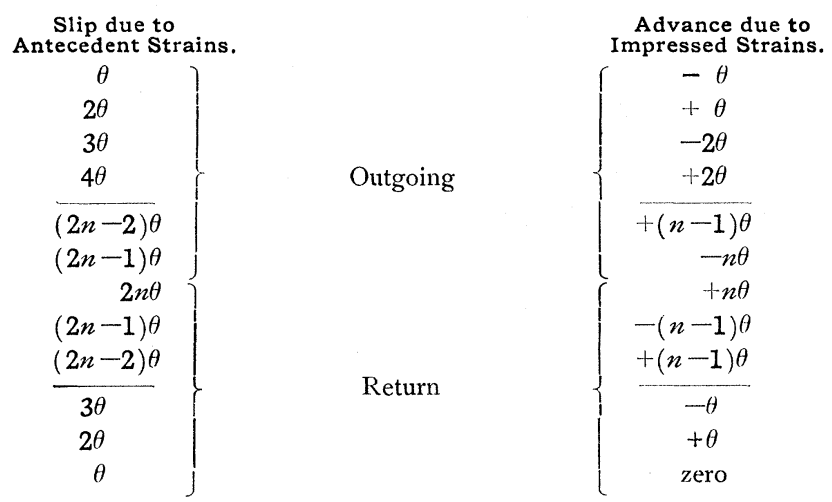

In the outgoing series, where the march of stress is increasing, the even antecedent twists are twice as large as the even (positive) impressed twists. In the odd twists, the former are always relatively less than this excess. Hence the slip in the even twists must be in excess of the slip in the odd twists, and thus the latter (negative) are given by peaks in the curve of rigidity, relatively to the valleys occupied by the former.

On returning the antecedent strains of the odd twists are the larger relatively to the corresponding (negative) twists in action. Hence the slip of the odd twists are relatively in excess and the even twists (positive) thus correspond to the peaks in the curve. 
There can be no doubt that this explanation of the alternations of curve $\mathrm{I}$ is fundamentally correct. On closer inspection, however, an apparant discrepancy is revealed in relation to the curves 2. For whereas the increments of rigidity increase indefinitely with $n \theta$, the limit of curve 3 being as yet far off, the slips reach a minimum very early, as indicated by the curves 2 in question. One would therefore expect the oscillations of the curves I to cease at the same early limit.

A key to the situation is already given by the curves 2, which become markedly oscillating after the limit in question has been reached, while the character of these oscillations is reproduced and accentuated in the curves $\mathrm{I}$, either in the same or the opposite phase. $^{1}$ After passing $\pm 40^{\circ}$ of impressed twist, the slip is picked up, as it were, by the concomitant advance of rigidity. Only a part of the total slip is manifest.

The probability of this state of things I have already put in evidence in the results of experiments in which slip in the absence of impressed strain was found to be a continually increasing quantity ${ }^{2}$ even beyond $360^{\circ}$.

Results for Successively Increasing, Asymmetrically AlterNATing Twists.

8. Results for Slip Alone.-Before proceeding to further details, therefore, it is necessary to study temporary set in detail, under circumstances in which there is antecedent twist, but no impressed or acting twist. There must then be slip without the increment of rigidity. This may be done in accordance with the following scheme :
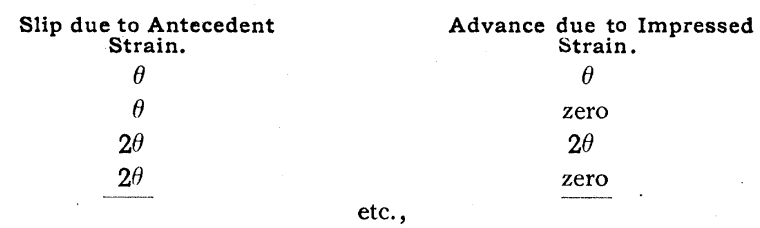

in which for the even twists the wire is brought back to the unstrained state. In other words the wire is to be twisted successively

1 The present discussion is in the main experimental and qualitative. A more detailed theoretical investigation will be given elsewhere.

2 American Jour. of Science, XI., I9r r, p. 105 et seq., $8 \& 8,9$. 


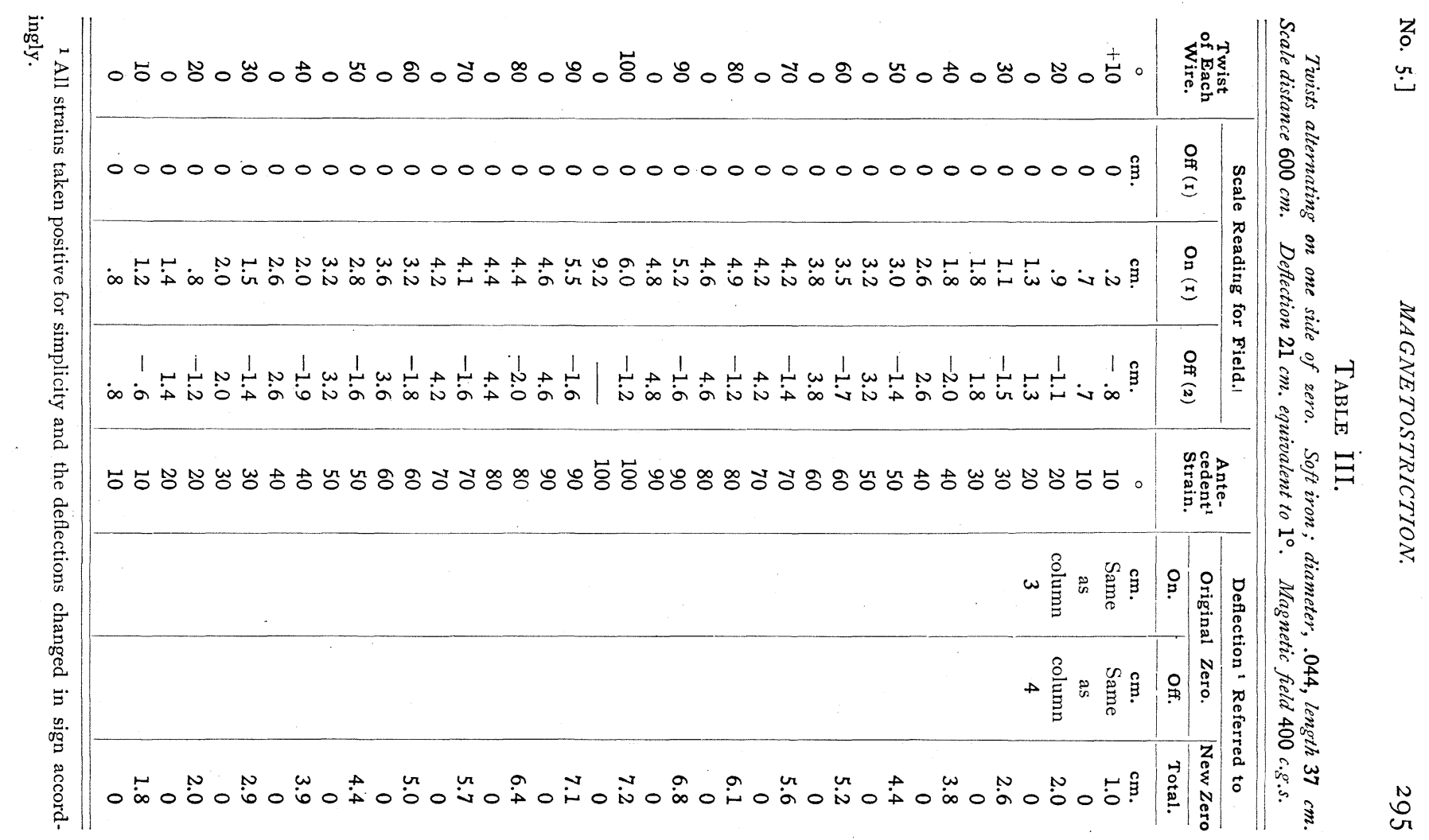


to $n \theta$, magnetized during the action of the impressed torque with a record of the scale reading before and after magnetization; then untwisted and a similar record made. Results obtained in this manner are given in Table III. in the usual way. The table consists of two parts, of which the first is the outgoing series of increasing twists, each successively brought back to zero and the temporary

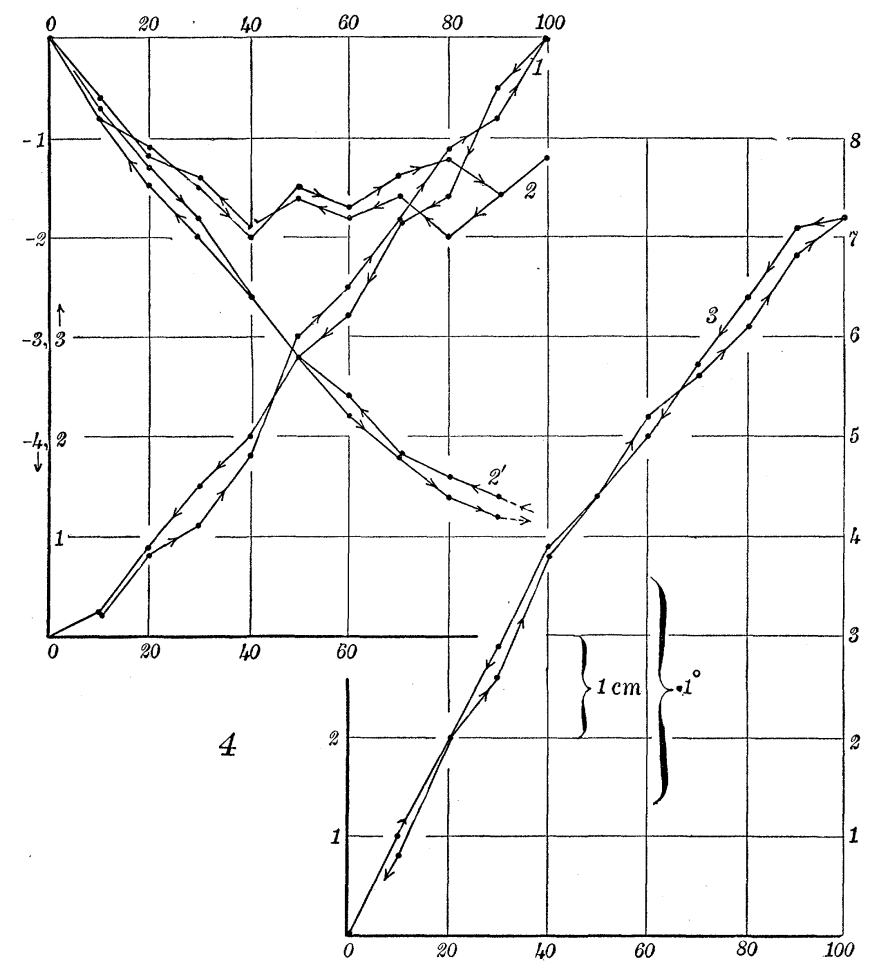

Fig. 4.

set shaken out by magnetization. The second part is the return series of corresponding data. The helix was provided with an internal water jacket to obviate changes of temperature, though continuous effects of this kind (ordinary viscosity) would be eliminated without this precaution. As a whole the two series are coincident, since the difference of a few millimeters in a scale distance of six meters can hardly be vouched for in experiments like the present, 
where there is inevitably much manual interference in twisting and untwisting.

The graph (Fig. 4) for this table is constructed in the usual way, either the antecedent $t$ wists or the impressed twists being the abscissas and the deflections due to changes of rigidity, the ordinates. Curve I shows the superposed effect of slip and advance in the presence of magnetic field and of impressed strain, the notation following the above. The curve should be smooth; it is not quite so for reasons presently to be stated. There is a gap due to curtailed ordinates below $40^{\circ}$ and resulting from excess of slip in this region. Above $40^{\circ}$, the mean line is nearly straight, and if prolonged backwards would nearly intersect the origin. The slope $d n / n=2 d \theta / \theta=.003$. Direct and return curves are practically identical. The curve shows a radical departure from the corresponding case (I) in Fig. 3, as predicted.

In curve 2 , showing the temporary set in the presence of the impressed strain stated, the oscillations begin markedly at about $40^{\circ}$, as usual, in spite of the totally new schedule of operations. The alternations of curve I are imaged in the same phase with accentuation throughout, the reverse having been the case above. The direct and retrograde curves are not coincident throughout. The slope is about $d \theta / \theta=.003$.

Curve $2^{\prime}$ is the specially new feature of the present experiments. It shows the amount of temporary set or slip for any antecedent strain in the absence of impressed strain. The smooth curve obtained in this case, practically coincident in the outgoing and the return branches, is evidence of the truth of the inferences expressed in the last paragraph. The curve also comes to a limit eventually, but the limit is considerably beyond the limits of observation. When contrasted with curve 2 , the difference in ordinates shows the amount of slip picked up by the magnetically incremented rigidity, for any angle of twist. The initial slopes of 2 and $2^{\prime}$ are about identical, $d \theta / \theta=.003$, having about the same value with the sign changed as in curve I. This curious feature is noteworthy as it occurs again below.

Curve 3 has the usual meaning, being the advance due to the magnetically increased rigidity in the absence of slip. The initial 
coefficient here is about $d n / n=2 d \theta / \theta=.0092$, the usual value. The curve happens to consist of two nearly linear parts, one between $0^{\circ}$ and $40^{\circ}$, the other between $40^{\circ}$ and $90^{\circ}$, corresponding to the oscillations of curve 2 .

Looking over the whole series of phenomena, one derives the impression that the effects are not quite statistical, $i$. e., that the number of molecules immediately engaged are not so numerous as in the kinetic theory of gases, for instance, and that for this reason the phenomena fall short of obedience to sharp laws. Sufficient variation was made in the apparatus to throw out errors due to it; but whether the mechanical interference which must inevitably accompany these experiments will not also shake out temporary set, remains to be seen.

\section{TABLE IV.}

Twists alternating on one side of zero. Magnetic field 800 c.g.s., soft iron, diameter .024 , length $35 \mathrm{~cm}$., scale distance $500 \mathrm{~cm}$., $18 \mathrm{~cm}$. equivalent to $1^{\circ}$, nearly.

\begin{tabular}{|c|c|c|c|c|c|}
\hline \multirow{2}{*}{$\begin{array}{c}\text { Twist of } \\
\text { each Wire. }\end{array}$} & \multicolumn{3}{|c|}{ Scale Reading when Field is } & \multirow{2}{*}{$\begin{array}{l}\text { Antecedent } \\
\text { Strain. }\end{array}$} & \multirow{2}{*}{$\begin{array}{c}\text { Total } \\
\text { Deflection. }\end{array}$} \\
\hline & Off $(x)$ & On $(x)$ & Off (2) & & \\
\hline 0 & $\mathrm{~cm}$ & $\mathrm{~cm}$ & $\mathrm{~cm}$ & $\circ$ & $\mathrm{cm}$. \\
\hline-360 & 0 & 9 & -7 & 360 & 16 \\
\hline 0 & 0 & 9 & 9 & "6 & 0 \\
\hline+360 & 0 & -11 & 5 & ، & 16 \\
\hline 0 & 0 & -10 & -10 & “" & 0 \\
\hline-360 & 0 & 12 & -4 & “ & 16 \\
\hline 0 & 0 & 10 & 10 & “ & 0 \\
\hline+360 & 0 & -12 & 5 & “ & 17 \\
\hline 0 & 0 & -10 & -10 & “ & 0 \\
\hline-180 & 0 & 6 & -6 & 180 & 12 \\
\hline 0 & 0 & 9 & 8 & "6 & 0 \\
\hline+180 & 0 & -6 & 7 & " & 13 \\
\hline 0 & 0 & -8 & -8 & "6 & 0 \\
\hline-180 & 0 & 6 & -5 & $" 6$ & 11 \\
\hline 0 & 0 & 8 & 8 & "6 & 0 \\
\hline+180 & 0 & -6 & 5 & $" 6$ & 11 \\
\hline 0 & 0 & -8 & -8 & " & 0 \\
\hline-90 & 0 & 2 & -5 & 90 & 7 \\
\hline 0 & 0 & 5 & 5 & " & 0 \\
\hline+90 & 0 & -3 & 4 & " & 7 \\
\hline 0 & 0 & -5 & -5 & " & 0 \\
\hline-90 & 0 & 2 & -5 & $" 6$ & 7 \\
\hline 0 & 0 & 5 & 5 & " & 0 \\
\hline+90 & 0 & -2 & 4 & 6 & 6 \\
\hline 0 & 0 & -6 & 0 & " & 0 \\
\hline
\end{tabular}

${ }^{1}$ Strain taken positive for simplicity and deflections changed in sign accordingly. 
9. Case of Larger Twists. Thin Wire.-The following data for a thinner wire are taken from my earlier work where they occur in a different connection and without being numerically stated. Readings were made to centimeters, not to millimeters as above; but the wire being thinner, showed correspondingly larger deflections.

One may note that while the total deflection (increment of rigidity in the absence of slip) remains nearly constant for any twist, the ratio of the component slip and advance implied may change in marked degree. This is particularly noticeable for the twist of $360^{\circ}$.

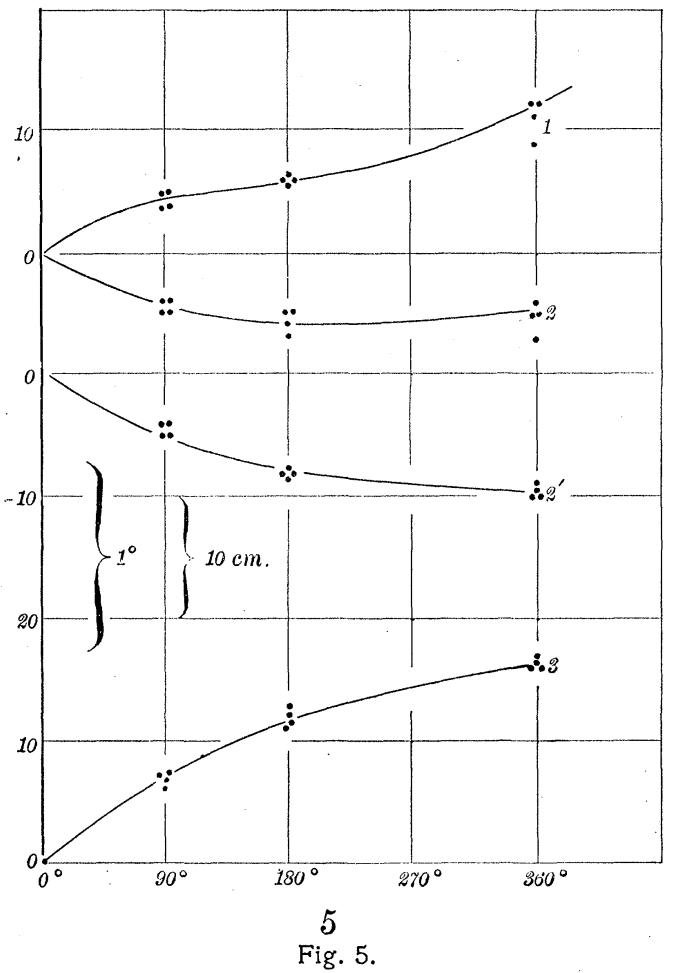

The graphs, Fig. 5, are numbered as in the preceding chart. Remembering that, in view of smaller diameters $(.024 \mathrm{~cm}$.), the twists here are relatively greater, results corroborating the preceding paragraph may be deduced. Curve 2 reaches a limit much before curve $2^{\prime}$, the difference being the slip picked up during 
magnetization by the coincident increment of rigidity. The initial slopes of curves I and $2(d \theta / \theta=.003)$, are nearly equal and opposite ; the initial slopes of 2 and $2^{\prime}$ nearly identical, showing the paramount initial (vanishing twists) importance of slip. Curve 3 and curve $2^{\prime}$ show similar contour, indicating close relation of slip and advance. The former is throughout numerically somewhat less than twice the latter, in ordinate. Initially, at least, the effect of magnetization may be conceived to be expended in producing slip and advance in the ratio of $I: 2$, though necessarily in opposed directions.

In Table IV. many alternations were made consecutively for each twist selected. This method does not lend itself well for the present purposes where a continuous record is wanted. Hence the following data were investigated.

\section{TABLE V.}

Twists alternating on one side of zero. Magnetic field 400 c.g.s., soft iron, diameter .044 , length $37 \mathrm{~cm}$., scale distance $600 \mathrm{~cm}$., deflection $21 \mathrm{~cm}$., equivalent to $1^{\circ}$.

\begin{tabular}{|c|c|c|c|c|c|}
\hline \multirow{2}{*}{$\begin{array}{c}\text { Twist of } \\
\text { each Wire. }\end{array}$} & \multicolumn{3}{|c|}{ Scale Reading for Field. } & \multirow{2}{*}{$\begin{array}{c}\text { Antecedent } \\
\text { Strain. } .^{1}\end{array}$} & \multirow{2}{*}{$\begin{array}{l}\text { Total Elastic } \\
\text { Deflection. }\end{array}$} \\
\hline & Off $(r)$ & On (I) & Off $(\mathbf{r})$ & & \\
\hline$\circ$ & $\mathrm{cm}$. & $\mathrm{cm}$. & $\mathrm{cm}$ & $\circ$ & $\mathrm{cm}$. \\
\hline 45 & 0 & 2.2 & -1.8 & 45 & 4.0 \\
\hline 0 & 0 & 3.5 & 3.5 & “ & 0 \\
\hline 90 & 0 & 5.1 & -1.3 & 90 & 6.3 \\
\hline 0 & 0 & 4.6 & 4.6 & 6 & 0 \\
\hline 135 & 0 & 7.3 & -1.1 & 135 & 8.4 \\
\hline 0 & 0 & 5.6 & 5.6 & “ & 0 \\
\hline 180 & 0 & 8.6 & -.8 & 180 & 9.4 \\
\hline 0 & 0 & 5.8 & 5.8 & “ & 0 \\
\hline 225 & 0 & $\left.\begin{array}{r}9.4 \\
10.0\end{array}\right\}$ & -.2 & $225^{2}$ & $\left.\begin{array}{r}9.6 \\
10.2\end{array}\right\}$ \\
\hline, 0 & 0 & 5.6 & 5.8 & " & -.2 \\
\hline 180 & 0 & 8.6 & -.9 & 180 & 9.5 \\
\hline 0 & 0 & 4.9 & 5.1 & "6 & -.2 \\
\hline 135 & 0 & 7.0 & -.9 & 135 & 7.9 \\
\hline 0 & 0 & 4.8 & 5.1 & " & -.3 \\
\hline 90 & 0 & 4.9 & -1.7 & 90 & 6.6 \\
\hline 0 & 0 & 4.3 & 4.5 & "6 & -.2 \\
\hline 45 & 0 & 1.6 & -2.0 & 45 & 3.6 \\
\hline 0 & 0 & 2.4 & 2.6 & " & -.2 \\
\hline
\end{tabular}

1 Strains taken positive and deflections changed in sign accordingly.

2 Permanent set occurs here. After final unlocking, the zero was at $-33 \mathrm{~cm}$., being a set of $1.6^{\circ}$. 
Io. Case of Larger Twists. Thicker Wire.-The observations of Table $\mathrm{V}$. were made for the same diameter and length of soft iron

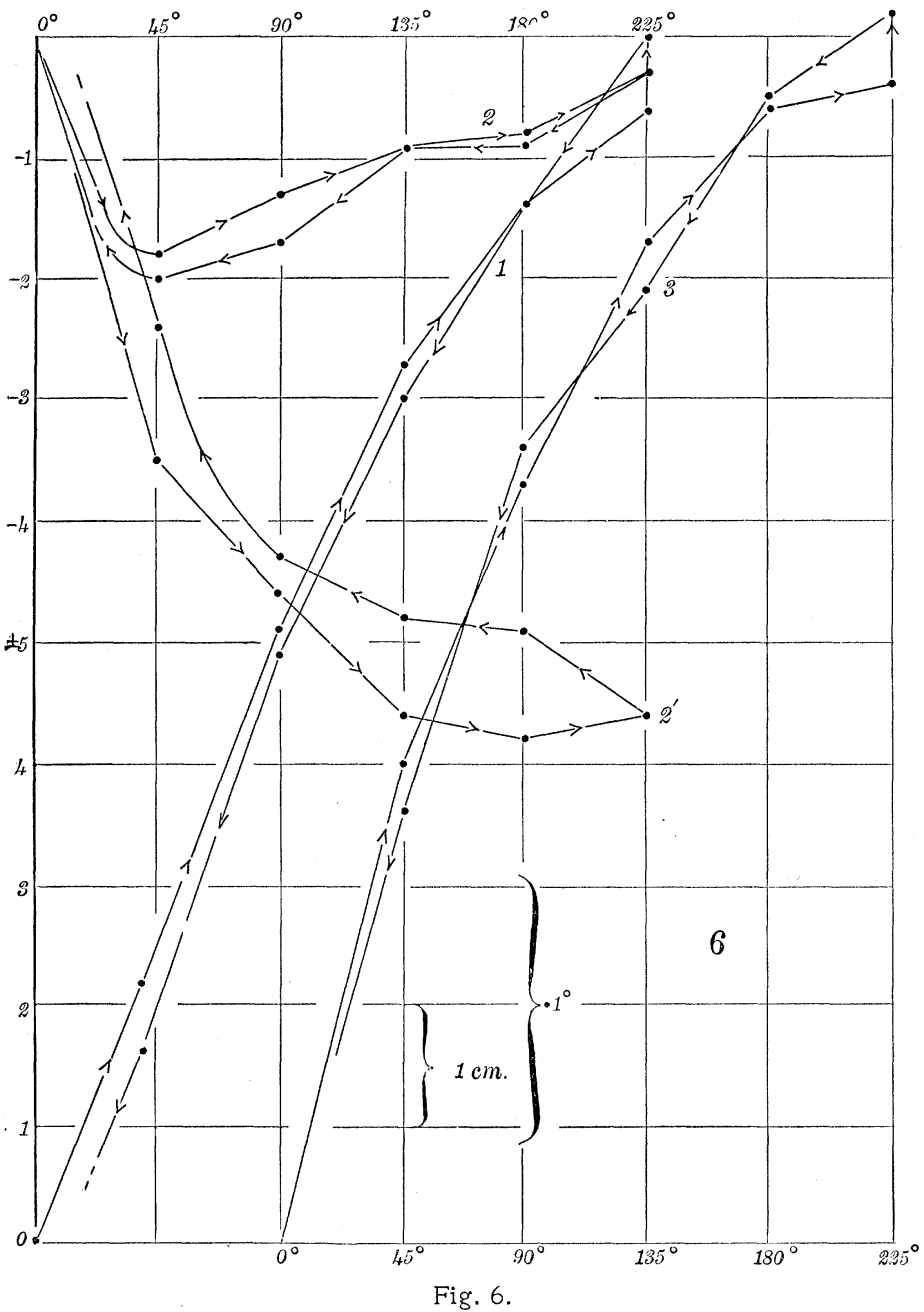

wire occurring throughout the bulk of the above experiments. It being the object to proceed quite up to the limits of elasticity, twists exceeding $200^{\circ}$ were necessary, and the data are given in the following table containing both an outgoing series and a return therefrom. The inevitable occurrence of permanent set here before $225^{\circ}$ is reached, interferes with coincidence between the two series. 
The results are given in the accompanying graph, Fig. 6, in the usual way, the abscissas being either antecedent strains or impressed strains as required. Curve $2^{\prime}$ shows the temporary set in the absence of impressed strain, curve 3 the advance due to magnetically increased rigidity in the absence of slip. In curve $I$ there is superposed slip and advance in the presence of impressed strain and of magnetization. In curve 2 , slip in the presence of impressed strain but absence of magnetization.

The smoothness of curve I is marked except at the end, where after the occurrence of permanent set, the direct and return curves are shifted laterally about $\mathrm{I}$ or 2 degrees relatively to each other. The initial slope is $d \theta / \theta=.0026$, somewhat smaller than above, but the initial curve is not so near the initial tangent. Greater smoothness corresponds to less interference, since the steps are $45^{\circ}$. In other respects curve I contains but little new information.

The case of curve 2, however, presents novelties. The above sharp minimum at about $40^{\circ}$ is substantiated, after which the curve in its progress toward $225^{\circ}$ nearly falls off to a vanishing deflection in the region of permanent set. The meandering path between $40^{\circ}$ and $100^{\circ}$ is again suggested by the new curve. The initial slope practically coincides with $2^{\prime}$.

Similarly definite results are obtained from curve $2^{\prime}$, showing the occurrence of slip alone in the absence of impressed strain. Temporary set reaches a definite limit at about $200^{\circ}$, after which there is unstable molecular equilibrium merging into permanent set. This lapse into a new and permanent molecular grouping which cannot again be transferred back by the magnetic shake-up, is the reason for divergence in the outgoing and the return series. The whole of the temporary set does not vanish, however, a natural result seeing that the yield in a practical case is always local and not distributed throughout the wire. The slope here is about $d \theta / \theta=.0037$, but it is not the initial tangent.

Curve 3, finally with an initial slope corresponding to

$$
d n / n=2 d \theta / \theta=.0082,
$$

which naturally falls short of the above value, rises to a definite limit with the occurrence of set. If this were obviated by much 
twisting, the limit would be more remote as is known from the earlier experiments.

I I. Conclusion and Summary.-Before proceeding to definite statements it is expedient to reduce all data to common torsion standards. In my earlier work ${ }^{1}$ the obliquity ( $\omega$ radians) of the external fiber was found to be convenient for reference, while all twists were expressed (in rates $\tau^{\circ}$ ) per unit of length. This to some extent eliminates the arbitrary lengths and radii involved. If the twist of each wire is $\theta^{\circ}, \tau=\theta / l$, where $l$ is the length of the wire of radius $r$. Moreover $\omega=\pi r \theta / \mathrm{r} 80 \times l=\pi r \tau / \mathrm{I} 80$.

For the two lengths and diameters occurring $(2 r=.044, l=37$ $\mathrm{cm}$., and $2 r=.024, l=35 \mathrm{~cm}$.), the constants, are therefore, $\omega=\mathrm{IO} \times \theta / \mathrm{IO}^{6}$ nearly, and $\omega=26 \times \theta / \mathrm{IO}^{6}$. Thus the limits of temporary set (curves $2^{\prime}$ ) in the cases of Tables IV. and V. occur at $\omega=.0022$ and $\omega=.0020$ radians, which are practically the same. In my old results operating with curve 3, permanent set occurred when $\omega=.003$ radians; but here the limit was pushed to the utmost verge by hardening the locally weak places by continued twisting. It is understood that $\omega$ refers to elastic deformations, no matter how much permanent set may have been previously imparted.

The minimum of curve 2, for temporary set in the presence of impressed strain and evoked by magnetization occurs for $\omega=.0004$ radians in case of Table $\mathrm{V}$. In Table IV. the steps are too large, so that it can be merely located below $\omega=.0008$ radians.

The chief result of the present paper is the detection of the growth conditions of temporary set, a latent strain distinguished from permanent set in that the former may be shaken out of the soft iron wire by molecular agitation. Temporary set $d \theta$ is an instantaneous accompaniment of any twist within the elastic limits; it begins with the zero of twist at an initial rate of about $d \theta / \theta=.0037$, increasing at a retarded rate to a superior limit when the (elastic) obliquity of the external fiber exceeds .002 radians. After this the wire begins to yield locally, gradually, acquiring permanent set. The new molecular condition is stable and can not be shaken out. In soft iron temporary set is made mani- 
fest as a slip in the absence of impressed strain on instantly applying a magnetic field following an antecedent strain. The limiting amount of temporary set which can be stored may be estimated as .0015 of the limiting twist within the elastic limits.

In the presence of impressed strain, a longitudinal magnetic field produces both magnetically increased rigidity and temporary set, as superposed phenomena. For small twists $\theta$ the rate is $d \theta / \theta=.003$ and positive. For larger angles the increase is gradually retarded but continues until permanent set is definitely established. Thus magnetically increased rigidity supervenes throughout, being initially about twice the slip. When the antecedent twist differs from the impressed twist, slip is to be associated with the former and advance with the latter and the statement of the result requires a detailed analysis. Cf. $\S \S 4-7$.

The slip in these instances is initially quite as large as in the absence of impressed strain, the initial coefficient being $d \theta / \theta=.0035$. It soon, however, reaches a pronounced minimum (algebraically), showing that slip is more and more arrested or picked up by the concomitant magnetically increased rigidity, until with the occurrence of permanent set, slip (evoked by magnetization in the presence of impressed strain) has all but vanished. The minimum in question is determined by $\omega=.0004$ to .0008 , while permanent set does not occur until $\omega=.002$ to .003 .

Finally magnetically increased rigidity in the necessary presence of impressed strain and the absence of slip increases with the impressed twist at an initial rate of $d \theta / \theta=.0046$. The coefficient of rigidity is thus of the order of $\mu=d n / n=. \mathrm{OI}$. As the twist increases the coefficient $\mu$ decreases to a limit of about .004, when $\omega$ exceeds .002, elastically. If the wire is hardened by indefinite twisting beyond the elastic limits, $\mu$ seems to approach a lower limit when $\omega$ is elastically .003. In my old results I thus found

$$
\mu=.002
$$

in a field of about 140 c.g.s. units and a large range of diameters $\left(.2\right.$ to $.02 \mathrm{~cm}$.). Mr. H. D. Day ${ }^{1}$ showed that the limit of $\mu$ in

\footnotetext{
${ }^{1}$ Day, American Journ. Sci., III., I 897 , p. 449. Cf. p. 456.
} 
very strong fields has not been reached, finding $\mu=.003, .005, .006$, in fields of 100,500 and I, 500 c.g.s. units, respectively.

The energy stored and eventually wasted in temporary set may be of passing interest. Since this work is half the product of the stress and the strain given by curves $2^{\prime}$, it appears that in case of Table V. $(2 r=.044)$ when slip has its largest value $\left(.27^{\circ}\right)$, this work does not exceed 300 ergs. Since the ratios of this work done in twisting and the energy wasted in slip are as the squares of the angles of twist and slip, about $2.4 / 10^{6}$ of the work done in twisting is lost in producing temporary set when the latter is at its maximum value just within the elastic limits. Initially the relative waste is much greater, being the square of the initial slope, $d \theta / \theta$, of curve $2^{\prime} ; i$. e., the waste is $(.0037)^{2}=14_{i}^{/} \mathrm{IO}^{6}$, nearly.

The case of Table IV. hardly contains sufficiently detailed data. About Ioo ergs would be stored up when the slip is at a maximum $\left(.5 \mathrm{I}^{\circ}\right)$ just within the elastic limits. The corresponding final waste ratio would be but $.4 / \mathrm{IO}^{6}$. The initial waste ratio is

$$
(.0033)^{2}=\mathrm{I} \mathrm{I} / \mathrm{IO}^{6}
$$

about of the same order as in the preceding case.

The energy wasted in magnetic hysteresis in double reversals per cubic $\mathrm{cm}$. of saturated soft iron is about 10,000 ergs. Comparing this with the energy wasted in temporary set, Table V. shows that $300 / \pi r^{2} l=\mathrm{I}, 300$ would be wasted per cubic $\mathrm{cm}$., and for a double reversal therefore 2,600 ergs.

From Table IV. it appears that $100 / \pi r^{2} l=1,590$, or about 3,200 ergs in a double reversal.

Seeing that it is somewhat difficult to get parallel cases for comparison, it will be noticed that the energy dissipated in a double reversal and saturated soft iron, magnetically in the one case, and mechanically in the other, is of a common order of values; for temporary set must be compared with residual magnetization.

Apart from its inherent importance as an introductory phase of permanent set, temporary set has therefore the additional interest of being a clearcut instance of purely mechanical hysteresis, without necessary relation to magnetism, as I understand it. To further establish this it will eventually be necessary to invent means to 
detect temporary set in other and non-magnetic metals. It follows moreover that the Weber-Ewing model of revoluble molecular magnets is either not the final resource for the physical explanation of magnetism, or else that its polar properties have a much wider application than simply to magnetic phenomena. On the whole the analogy of temporary set with magnetization is attractive ; magnetization may be shaken out of soft iron by mechanical tapping; similarly temporary may set be shaken out by magnetization, etc. In addition to the intimate connection of temporary set and magnetic hysteresis, it is also probable that the same slip lies at the root of Kelvin's "fatigue of elasticity," as yet an unclassified phenomenon in the domain of viscosity.

Brown University, Providence, R. I. 